\title{
Improved rapid transit network design model: considering transfer effects
}

\author{
Luis Cadarso ${ }^{1}$ • Ángel Marín ${ }^{2}$
}

\begin{abstract}
The rail rapid transit network design problem aims at locating train alignments and stations, maximizing demand coverage while competing with the current existing networks. We present a model formulation for computing tight bounds of the linear relaxation of the problem where transfers are also introduced. The number of transfers within a trip is a decisive attribute for attracting passengers: transferring is annoying and undesirable for passengers. We conduct computational experiments on different networks and show how we are able to solve more efficiently problems that have been already solved; sensitivity analysis on several model parameters are also performed so as to demonstrate the robustness of the new formulation.
\end{abstract}

Keywords Rail rapid transit · Network design · Transfers

\section{Introduction}

Increasing mobility and longer journeys caused by the growth of cities have stimulated the construction and expansion of rail rapid transit systems such as metro, urban rail, and light rail. These facts raise the concerns about energy constraints and greenhouse emissions. Because a network design heavily affects to its future operation and to the area where it is constructed, it is important to pay close attention to the future impacts on mobility and congestion.

This research was supported by Project Grants TRA2011-27791-C03-01 and TRA2014-52530-C3-1-P by the Ministerio de Economía y Competitividad, Spain.

Luis Cadarso

luis.cadarso@urjc.es

1 Departmental III Building, Rey Juan Carlos University, Camino del Molino s/n, 28943 Fuenlabrada, Spain

2 E.T.S. Aeronautical and Space Engineering, Technical University of Madrid, Pza. Cardenal Cisneros, 3, 28040 Madrid, Spain 
Considerable attention has been given to the utilization of passengers railway systems as a relative efficient and eco-friendly traffic mode. The strategic and tactical railway planning problems may be summarized by the two following steps: the railway network design problem and the line planning problem.

Designing a Rapid Transit Network (RTN), or even extending one that is already functioning, is a vital strategic subject due to the fact that it reduces the future traffic congestion, passenger travel time and pollution. The maximum coverage of the demand by the new network is the main goal. Location decisions are made considering a list of potential rapid transit corridors and stations, and considering budget availability. This problem is considered the strategic level, and it usually does not consider capacity.

The following step after designing a RTN is planning its lines (origin and destination stations, stops and frequencies). This problem is at a tactical planning level. It consists of designing a line system such that all travel demands are satisfied and certain objectives, such as maximizing the service towards the passengers and minimizing the operating costs of the railway system, are met. At this level the system capacity is considered.

A crucial part of the planning process is the underlying network design, which consists of two intertwined problems: location of stations and alignments between them. These design decisions are considered at an upper level and demand behavior at a lower level. At the upper level the maximum coverage of demand is usually the main objective, taking line and budget constraints into account. At the lower level demand behavior is modeled accounting for trip alternatives; here, the existing infrastructure and the new one to be constructed are considered. This choice may be modeled assuming that passengers choose the most convenient routes and modes to carry out their trips.

In public transport, a convenient attribute for attracting passengers is to offer direct trips without transfers. In our approach, transfers are penalized considering that passengers always try to avoid them when commuting. Previous research in RTN Design (RTND) usually do not consider passenger transfer costs. Some authors (see García et al. 2006 for example) have attempted to model transfer costs but their approaches have failed to solve medium- or large-scale problems.

This paper presents two different approaches to the RTND problem. The classic weak formulation, which provides a good approach to the problem; however, it is not a good modeling choice for solving large-scale problems. And a strong formulation, which allows to efficiently handle transfers in real-scale problems.

\subsection{State of the art}

Historically, network design research has focused first on determining a single alignment and the location of stations through it. Laporte et al. (2000) present a survey article that reviews the main available methods for network design problems.

Bruno et al. (2002) maximize the coverage of the demand locating a rapid transit line. Bruno et al. (1998) and Laporte et al. (2005) incorporate origin-destination demand data. Laporte et al. (2002) and Hamacher et al. (2001) deal with the problem of locating a set of stations on a given alignment.

Lately, Laporte et al. (2007) incorporate the station and track location problems in the previous network design models; they assume a set of possible alternatives to maximize the coverage of the demand and include budget constraints as side constraints. Marín (2007) studies the inclusion of free but bounded number of lines, where each line's origin and destination are freely chosen. Gutiérrez-Jarpa et al. (2013) present a rapid transit network design model to minimize travel cost and maximize traffic capture while segments within 
broad corridors to connect some vertex sets are built. Laporte et al. (2011a) study a robust formulation for the RTND problem; they implement constraints in order to provide a network with several alternative routes for given origin-destination pairs. Bruno and Laporte (2002) describe a visual interactive decision support system for simultaneous location of several rapid transit lines in a city. The system produces network configurations of the type selected by the user and computes a number of effectiveness measures. It can consistently produce several alternative solutions within short computing times.

RTND formulations are very complex. Hamacher et al. (2001) show that the problem of station location on a network is an NP-hard problem. Similarly, Laporte et al. (2011a) prove that the RTND problem formulation is an NP-hard problem. Because of the computational time required to solve RTND models, new solving approaches have been developed: Marín and Jaramillo (2008) propose an accelerated Benders Decomposition to reduce computational times. Escudero and Muñoz (2009) propose a two-stage approach for solving this problem. In the first stage, an integer model is solved for selecting the stations and the links between them to be constructed. In the second stage, the line design problem is solved by means of a procedure that assigns each selected link to exactly one line.

Laporte et al. (2011b) review some indexes for the quality of a rapid transit network, as well as mathematical models and heuristics that can be used to design networks. Because models for RTND problems are difficult to solve when the network size is increased, heuristic algorithms have been developed. Dufourd et al. (1996) apply the Tabu Search heuristic approach. Bruno et al. (2002) use other local searches that try to locate a new station compatible with the constraints at each iteration. Kermansshahi et al. (2010) develop a simulated annealing methodology to solve networks of medium-scale cities. Fan et al. (2006) use Simulated Annealing to solve a transit network design problem. Ngamchai and Lovell (2003) and Tom and Mohan (2003) use Genetic Algorithms to solve the same type of problems.

The use of an adequate formulation is of vital importance to efficiently solve models in integer programming. Gendron and Crainic (1994) apply the classical relaxation methods to several formulations of a fixed charge multicommodity capacitated network design problem. They compare theoretically and computationally the relative strength of the relaxations. Gendron et al. (1999) study several formulations for the capacitated network design model taking into account the quality of the lower bounds that can be generated through relaxations as well as the methods used to solve the model.

The number of intermediate stations for each trip may be an important attribute for passengers. Repolho et al. (2013) present a mixed-integer optimization model that determines the optimal location (and number) of stations along a railway line that will be introduced over an existing transportation network. The model takes into account the sensitivity of rail ridership to time losses because of stops at intermediate stations, as well as (static) competition from other modes. Furthermore, a decisive attribute for attracting passengers to the public mode is to offer direct trips without transfers. Transferring is annoying and undesirable for passengers. The previous references consider travel cost as the time spent in traveling (or distance traveled) without accounting for any transfer cost. Some authors study the network design problem considering transfers between different lines. García et al. (2006), Ngamchai and Lovell (2003) and Zhao and Ubaka (2004) study the problem making some assumptions about the speed of the trains and frequency of the services. These authors fail to solve realscale network design problems. Moreover, data such as service frequency and speed of the trains are highly uncertain at a strategic level, and this uncertainty is not addressed in the presented modeling approaches. 


\section{Contributions}

In this paper, we present a mixed integer non-linear programming model for the rapid transit network design problem that improves previous rapid transit network design formulations.

We use a formulation for the rapid transit network design problem, with a strong linear programming relaxation, where transfers are introduced. The number of transfers in a trip is a decisive attribute for attracting passengers to the new network: transferring is annoying and undesirable for passengers. Therefore, the network design must account for transfers in order to provide a better service and attract more passengers. Previous works, such as García et al. (2006), have considered transfers. However, the formulations developed before were only able to handle small-scale problems. Here, we develop a formulation which is suitable to deal with real-scale problems.

In order to demonstrate our formulation robustness we make sensitivity analysis on several model parameters in case studies based on realistic networks. Our experimental results show that we are able to solve problems where other works failed: we make computational comparisons between our approach and previous approaches.

\section{Outline of the paper}

The remainder of this paper is organized as follows. In Sect. 2 we describe the rapid transit network design problem. Section 3 presents our improved modeling approach. In Sect. 4, computational experiments for a real-world problem using real data are described. Section 5 concludes with a discussion of major findings.

\section{Problem description}

In this section, the RTND problem is introduced. First, we describe the rapid transit infrastructure. Next, we explain how we model passenger demand.

\subsection{Rapid transit infrastructure}

The rail rapid transit network consists of potential ares and nodes. We have two different types of nodes: centroids and stations. Centroid nodes are those where the demand is generated or attracted to. Station nodes are those where the network is built on and where passenger demand enters the network. We assume that the location of the potential stations is given. Then, we model the infrastructure as a graph with nodes $i \in N$, and with the set $A$ of feasible (bidirectional) arcs linking them. Each potential station $i$ has an associated construction cost $c_{i}$ and each arc $i j \in A$ a pair $\left(c_{i j}, d_{i j}\right)$ of weights: the construction $\operatorname{cost} c_{i j}$ and the distance $d_{i j}$.

The arcs are used to define the new lines to be constructed. $L$ is the new line set. These lines must be understood as elements to support the design, but not as services with frequencies, because we study an uncapacitated network design problem.

As we are studying a real life problem, the new infrastructure to be constructed will not be isolated from the current network. We will consider the existence of a current transport network formed by different mode alternatives such as private cars and buses. This current network may have stations in common with the rapid transit network to be constructed. However, they are independent. 


\subsection{Passenger demand}

The demand is characterized by an origin and a destination. We assume that the mobility patterns in a metropolitan area are known. This implies that the number of potential passengers from each origin to each destination is known. We define passenger groups as follows: $w=\left(o(w), d(w), g_{w}\right)$, where $o(w) \in N$ is the origin centroid, $d(w) \in N$ the destination centroid, and $g_{w}$ is the passenger group size (i.e., number of passengers of a given group $w$ ).

The demand will be realized through available paths in the new network or trough a path within the current network. Each passenger group $w \in W$ will choose a path. Each path is characterized by its origin, destination and the arcs belonging to it. The demand will choose its path based on the generalized travel cost. We assume the generalized cost is given by two different components:

1. the distance each passenger group must travel to reach its destination. This distance is equal to the sum of all the arcs' distances $\left(d_{i j}\right)$ in the path for the new network;

2. and the transfer cost. Because passengers choose the most convenient routes and modes in order to carry out their trips, a decisive factor for attracting passengers to the new network is to offer direct trips without transfers. In strategic planning, a planning horizon of several years is available and the details related to the operative level, such as frequencies of the lines and timetable, are not known. Therefore, the transfer cost will be a penalty in terms of distance.

Passenger behavior is modeled by comparison of the current and the new generalized costs. In the case of the current network, the generalized cost $u_{c u r}^{w}$ for each passenger group $w$ is assumed to be known. We acknowledge that generalized costs for the current network are not well known because the problem we study is at a strategic planning level. Therefore, congestion is an important issue that must be accounted for. Although the distance to be traveled by passengers is independent of congestion, travel time is highly dependent on it. We introduce a congestion parameter $\mu_{w}$ for each passenger group $w$ which accounts for different scenarios of congestion in the current network in order to adjust the generalized $\operatorname{cost} u_{c u r}^{w}$.

\section{Assumptions}

The model assumes the Wardrop's First Principle. This principle affirms that users choose their route according to the least expensive trip. The mathematical formula for this principle gives rise to equilibrium models in networks. The principle has been formulated via optimization problems, variational inequalities or non-linear slackness conditions. Therefore, the resultant RTND models tend towards a mathematical programming structure of huge complexity; we have avoided the direct appearance of these constraints in our approximation. This choice is coherent with the assumption that the system capacity is not considered in the model.

The effect of congestion is only considered in the current generalized cost, which corresponds to the current network. Because congestion could also decrease in the current network when the new network is constructed, we study decreases and increases in the current network's congestion level. This paper does not consider line frequency planning of the new services to be defined within the new network, and therefore it is reasonable to consider that non-congested networks are being designed. This is also consistent with the fact that we study rapid transit systems, where there is usually an isolated infrastructure for the transport system (e.g., metro and rapid bus systems). We acknowledge that the level of 
passenger demand can influence dwell times; however, we neglect this effect because we do not consider system's passenger capacity. Therefore, generalized costs in the new network are assumed to be independent of the level of congestion. Then, the cost of the physical network to be constructed is independent of the level of congestion in the new network, but the infrastructure construction depends on the congestion of the current network through modal split constraints.

Passengers use the new network considering transport lines, but the choice of a route does not determine the line that the passenger uses. The passenger him/herself may even use the same route with different lines (strategy). A tactical planning model must take this fact into account. The network design criteria force the user to choose the least costly route. This fact, assuming also a non-congested new network, allows us to model Wardrops principle in a simplified way.

We do not consider combined trips in this work. This is due to the fact that the type of systems we study, i.e., rapid transit systems such as the ones in Cadarso and Marín (2010, 2011, 2012), are generally used once by passengers. This is, it is rare the case where a passenger enters the rapid transit system, changes to another transport system, and again comes back to the rapid transit system to reach his/her destination. However, the extension of the formulation presented in Sect. 3 in order to include combined trips is straightforward: arcs representing the current network can be added to $A_{r}$, which is the subset of arcs linking the potential stations, which would have no construction cost and an increased distance value to represent the extra cost incurred due to the use of a different transport system.

\section{Rapid transit network design model}

The Transfers RTND Model (TRTNDM) is a non-linear mixed integer programming model. It aims at computing a network design in order to decide at which nodes are the stations to be located so as to attract as many passengers as possible to the new network. Since resources are limited, budget constraint on construction cost is imposed.

The model presented here minimizes a combination of system-related and service-related criteria subject to constraints for the underlying rapid transit network design and passenger use problems. The purpose of the constraints is summarized as follows:

- Regarding the rapid transit network:

1. a budget limit is enforced;

2. arcs are located if and only if its origin and destination stations are located;

3. lines do not form cycles;

4. and the number of arcs and stations in each line is limited.

- Regarding passenger demand:

1. the demand choice is modeled according to the all-or-nothing principle;

2. and the demand follows a feasible path on either the new or current network.

- Finally, passenger demand is allocated either to the located infrastructure or to the current network. 
Description of the sets, parameters and variables used in the mathematical formulation follows.

\section{Sets}

$-N$ is the set of nodes indexed by $i$ and $j$.

$-N_{c}$ is the subset of centroids.

$-N_{r}$ is the subset of potential stations.

- $A$ is the set of arcs (bidirectional) linking the nodes. It is indexed by $i j$ (its origin node $i$ and its destination node $j$ ).

- $A_{r}$ is the subset of arcs linking the potential stations.

- $A_{o}$ is the subset of dummy arcs between origin centroids and any potential station.

- $A_{d}$ is the subset of dummy arcs between potential stations and every destination centroid.

- $A_{f}$ is the subset of fictitious arcs between any origin-destination pair corresponding to current network.

- $N(i)=\left\{j: \exists i j \in A_{r} \cup A_{d} \cup A_{o}\right\}$ is the subset of nodes adjacent to node $i$.

- $N_{r}(i)=\left\{j: \exists j \in N_{r} \cap N(i)\right\}$ is the subset of potential stations adjacent to node $i$.

- $W$ is the set of passenger groups indexed by $w$. It is defined by an origin centroid $(o(w))$ and a destination centroid $(d(w))$.

$-L$ is the set of lines.

\section{Parameters}

$-d_{i j}$ is the length of each arc $i j$. The length of the arcs usually corresponds to the euclidean distance between the nodes if the system is underground and the street distance if it is at ground. However, forbidden regions will increase the distance.

- $g_{w}$ is the number of passengers in passenger group $w=(o(w), d(w))$.

$-c_{i j}$ is the cost of constructing an are $i j$.

$-c_{i}$ is the cost of constructing a station $i$.

$-c_{\max }$ is the upper budget bound.

- $\mu_{w}$ is the congestion factor for each passenger group $w$ in the current network.

- $v_{w}$ is the transfer cost per transfer and passenger group $w$ in the new network.

- $u_{c u r}^{w}$ generalized cost for passenger group $w$ in the current network.

- $o_{i}^{w}$ is 1 if passenger group $w$ enters the network at centroid $i$, and 0 otherwise.

$-d_{i}^{w}$ is 1 if passenger group $w$ leaves the network at node $i$, and 0 otherwise.

$-\alpha$ is the weight in the objective function for the number of passengers using the current network.

$-\beta$ is the weight in the objective function for location costs.

$-\gamma$ is the weight in the objective function for routing costs.

- $M_{\tau}$ is an upper bound (big enough) to the number of arcs per line traveled by passengers.

- $M_{l}$ is an upper bound to the number of arcs in the lines.

\section{Variables}

- $x_{i j}^{l} \in\{0,1\}$ is 1 if line $l \in L$ is located using the arc $i j \in A_{r}$, and 0 otherwise.

- $\psi_{i} \in\{0,1\}$ is 1 if potential station $i \in N_{r}$ is located, and 0 otherwise.

$-y_{i}^{l} \in\{0,1\}$ is 1 if line $l \in L$ is located using the potential station $i \in N_{r}$, and 0 otherwise.

- $h_{l} \in\{0,1\}$ is 1 if line $l \in L$ has at least one arc, and 0 otherwise. 
$-u_{w}^{n e w} \in \mathcal{R}^{+}$is the generalized cost in the new network for passenger group $w \in W$. Note that it depends on $f_{i j}^{w}, \tau_{w}^{l}$ and $\vartheta_{w}$ which are variables of the mathematical model.

- $f_{i j}^{w} \in\{0,1\}$ is 1 if passenger group $w \in W$ uses arc $i j \in A_{r} \cup A_{d} \cup A_{o}$ in the new network, and 0 otherwise.

- $f_{\text {cur }}^{w} \in\{0,1\}$ is 1 if and only if passenger group $w \in W$ uses the current network, and 0 otherwise. It denotes the use of the fictitious arc $i j \in A_{f}$.

$-\vartheta_{w} \in\{0,1\}$ is 1 if passenger group $w \in W$ uses the new network, and 0 otherwise.

- $\tau_{w}^{l} \in\{0,1\}$ is 1 if passenger group $w \in W$ uses line $l \in L$, and 0 otherwise. Consequently, the number of transfers a passenger group $w$ must perform is $\sum_{l \in L} \tau_{w}^{l}-\vartheta_{w}$.

\section{Mathematical formulation}

The TRTND model formulation is defined as the minimization of the number of passengers using the current network, location costs and routing costs. Hence, we are dealing with a multiobjective optimization model. As usual, we minimize a positive, linear combination of the different costs:

$$
\begin{aligned}
& \min z=\alpha z_{\text {cur }}+\beta z_{\text {loc }}+\gamma z_{\text {route }}, \\
& z_{c u r}=\sum_{w \in W} g_{w} f_{c u r}^{w}, \\
& z_{l o c}=\sum_{l \in L} \sum_{i j \in A_{r}, i<j} c_{i j} x_{i j}^{l}+\sum_{i \in N_{r}} c_{i} \psi_{i}, \\
& z_{\text {route }}=\sum_{w \in W} u_{w}^{n e w}+\mu_{w} u_{w}^{c u r} f_{c u r}^{w} . \\
& u_{w}^{n e w}=\sum_{i j \in A_{r} \cup A_{d} \cup A_{o}} d_{i j} f_{i j}^{w}+v_{w}\left(\sum_{l \in L} \tau_{w}^{l}-\vartheta_{w}\right) \quad \forall w \in W \\
& z_{\text {loc }} \leq c_{\max } \\
& x_{i j}^{l} \leq y_{i}^{l} \quad \forall i j \in A_{r}: i<j, \forall l \in L \\
& x_{i j}^{l} \leq y_{j}^{l} \quad \forall i j \in A_{r}: i<j, \forall l \in L \\
& x_{i j}^{l}=x_{j i}^{l} \quad \forall i j \in A_{r}: i<j, \forall l \in L \\
& y_{i}^{l} \leq \psi_{i} \quad \forall i \in N_{r}, \forall l \in L \\
& \sum_{j \in N_{r}(i): i<j} x_{i j}^{l}+\sum_{j \in N_{r}(i): i<j} x_{j i}^{l} \leq 2 \quad \forall i \in N_{r}, \forall l \in L \\
& \sum_{i j \in B: i<j} x_{i j}^{l} \leq|B|-1 \quad \forall l \in L, \forall B \subset N_{r},|B| \geq 2 \\
& \sum_{i j \in A_{p}: i<j} x_{i j}^{l} \leq M_{l} h_{l} \quad \forall l \in L \\
& \sum_{i j \in A_{r}: i<j} x_{i j}^{l} \geq h_{l} \quad \forall l \in L \\
& h_{l}+\sum_{i j \in A_{r}: i<j} x_{i j}^{l}=\sum_{i \in N_{r}} y_{i}^{l} \quad \forall l \in L \\
& u_{w}^{n e w} \leq \mu_{w} u_{c u r}^{w}\left(1-f_{c u r}^{w}\right) \quad \forall w \in W
\end{aligned}
$$




$$
\begin{gathered}
f_{i j}^{w}+f_{j i}^{w} \leq \sum_{l \in L} x_{i j}^{l} \quad \forall i j \in A_{r}: i<j, \forall w \in W \\
f_{o(w) j}^{w} \leq \sum_{l \in L} y_{j}^{l} \quad \forall j \in N_{r}, \forall w \in W \\
f_{i d(w)}^{w} \leq \sum_{l \in L} y_{i}^{l} \quad \forall i \in N_{r}, \forall w \in W \\
\sum_{k \in N(i)} f_{k i}^{w}-\sum_{j \in N(i)} f_{i j}^{w}=-o_{i}^{w}+d_{i}^{w} \quad \forall i \in N, w \in W \\
M_{\tau} \tau_{w}^{l} \geq \sum_{i j \in A_{r}} f_{i j}^{w} x_{i j}^{l} \quad \forall w \in W, l \in L \\
\vartheta_{w} \geq \tau_{w}^{l} \quad \forall w \in W, l \in L
\end{gathered}
$$

The minimization of trips in the current network is the main component of the objective function: the term in Eq. (2) accounts for it. The location cost in Eq. (3) is also minimized in order to avoid the construction of inoperative parts of network. Finally, the sum of the demand routing costs per passenger group is minimized through $\mathrm{Eq}$. (4). The generalized cost in the new network for passenger group $w\left(u_{w}^{\text {new }}\right)$ is given by (5). Variable $\vartheta_{w}$ indicates whether passenger group $w$ uses the new network. If it uses it, $\vartheta_{w}$ is 1 , and 0 otherwise. When $\vartheta_{w}$ is $1, u_{w}^{\text {new }}$ is the sum of the distances traveled by passenger group $w$ and the cost of transfers, $v_{w}$ times the number of transfers. Constraint (6) imposes the maximum available budget for constructing the new network. Constraints (7)-(10) are line location constraints. They are included in order to ensure that the arcs are not located if their origin and destination nodes are not previously located, and to ensure that for every located arc both riding directions are available. Constraints (11)-(12) are line path constraints. They guarantee that the lines follow a path without cycles. $B$ represents all the subsets of arcs in a path which form a cycle. Constraints (13)-(15) are line constraints. It must hold that $h_{l}=1$ if there is at least one arc associated to line $l$. Moreover, lines cannot have a number of stations greater than the number of located arcs plus one. Constraints (16) determine for each passenger group $w$ the modal split between the new and current network. Constraints (17) guarantee that each passenger group $w$ uses an arc only if this arc belongs to a line that has been constructed in the new RTN. Constraints (18)-(19) guarantee that each passenger group $w$ is routed from a centroid to a station, or from a station to a centroid only if the station belongs to a line that has been constructed in the new RTN. Constraints (20) are flow conservation constraints for each passenger group and at each centroid. They state that every passenger group must enter (in the origin centroid), leave (in the destination centroid) or continue (in the rest of nodes) in the network. Constraints (21) are used to count the number of lines each passenger group $w$ uses in the new network: each time a passenger group $w$ uses a line $l$, constraints (21) ensure that $\tau_{w}^{l}$ equals to 1 . Finally, constraints (22) determine the value for $\vartheta_{w}$ variables.

\section{Linearising and strengthening the formulation}

Constraints (21) are non-linear. However, they may be linearized using constraints (23).

$$
\tau_{w}^{l} \geq 1-\left(1-x_{i j}^{l}\right)-\left(1-f_{i j}^{w}\right) \quad \forall i j \in A_{r}, w \in W, l \in L
$$

The mathematical model presented above and the classic rapid transit network design model (see García et al. 2006; Marín and García 2007; Marín 2007) have a weak linear 
programming (LP) relaxation. Consequently the Branch \& Bound algorithm performance is poor. In order to improve the LP relaxation value, the formulation of the TRTND model is updated as follows: the term $\sum_{l \in L} x_{i j}^{l}$ is substituted by a single variable $\chi_{i j} \in\{0,1\}$ which determines whether the are is located or not. Similarly, $\sum_{l \in L} y_{i}^{l}$ is substituted by $\psi_{i}$. Then, the new topological variables are associated to the existence of the arcs, independent of line existence, and they are defined as:

- $\chi_{i j} \in\{0,1\}$ is 1 if arc $i j$ is located, and 0 otherwise.

The new constraints are given by:

$$
\begin{aligned}
& f_{i j}^{w}+f_{j i}^{w} \leq \chi_{i j} \quad \forall i j \in A_{r}: i<j, \forall w \in W \\
& f_{o(w) j}^{w} \leq \psi_{j} \quad \forall j \in N_{r}, \forall w \in W \\
& f_{i d(w)}^{w} \leq \psi_{i} \quad \forall i \in N_{r}, \forall w \in W \\
& \chi_{i j}=\sum_{l \in L} x_{i j}^{l} \quad \forall i j \in A_{r}: i<j
\end{aligned}
$$

Constraints (24)-(26) replace constraints (17)-(19). Constraints (27) link the new arclocation variables with the arc-location variables in the classic model. These previous constraints force us to introduce the following new set of constraints which ensures that the location of nodes and arcs is coherent:

$$
\begin{array}{ll}
\chi_{i j}=\chi_{j i} & \forall i j \in A_{r}: i<j \\
\chi_{i j} \leq \psi_{i} & \forall i \in N_{r}, \forall i j \in A_{r}: i<j \\
\chi_{i j} \leq \psi_{j} & \forall j \in N_{r}, \forall i j \in A_{r}: i<j
\end{array}
$$

Moreover, the objective function must be also updated. Equation (3) is substituted by Eq. (31).

$$
z_{l o c}=\sum_{i j \in A_{r}, i<j} c_{i j} \chi_{i j}+\sum_{i \in N_{r}} c_{i} \psi_{i},
$$

Therefore, we define the Improved TRTNDM (ITRTNDM) as the mathematical model given by (1)-(2), (31), (4)-(16), (24)-(30), (20), (23) and (22). Also, in order to compare the performance of the formulation presented here with previous formulations (i.e., the mathematical model in Marín and García 2007 and Marín 2007), we define the Improved RTNDM (IRTNDM) as the mathematical model given by (1)-(2), (31), (4)-(16), (24)-(30) and (20). In addition, the term $v_{w}\left(\sum_{l \in L} \tau_{w}^{l}-\vartheta_{w}\right)$ in constraints (5) is not considered in the IRTNDM.

\section{Case study and results}

We evaluate our model performance with case studies focusing on two different networks. One of the networks has been already defined and tested by other authors in the state of the art (see Laporte et al. 2007; Marín 2007). This network is denoted by R1 and has 9 potential stations, 30 arcs, 72 passenger groups and a total demand of 1044 passengers. Each potential station $i$ in network R1 has an associated construction cost $c_{i}$ and each arc $i j$ a pair $\left(c_{i j}, d_{i j}\right)$ of weights: the construction cost $c_{i j}$ and the distance $d_{i j}$. Figure 1 depicts Network R1. 


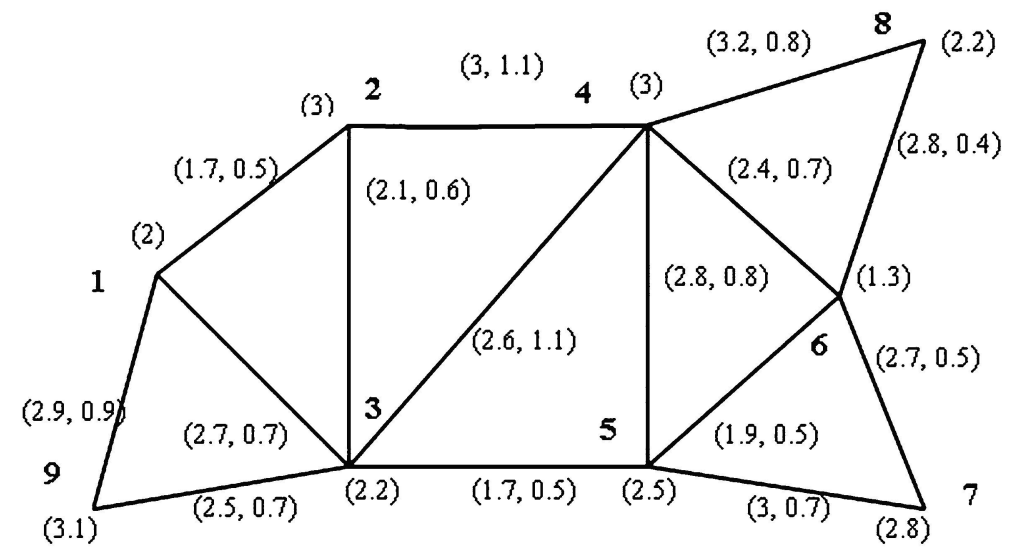

Fig. 1 Network R1

In network R1, the origin-destination demand $g_{w}$ for each passenger group $w \in W$ is defined by the following matrix:

$$
G=\left(\begin{array}{ccccccccc}
- & 9 & 26 & 19 & 13 & 12 & 13 & 8 & 11 \\
11 & - & 14 & 26 & 7 & 18 & 3 & 6 & 12 \\
30 & 19 & - & 30 & 24 & 8 & 15 & 12 & 5 \\
21 & 9 & 11 & - & 22 & 16 & 25 & 21 & 23 \\
14 & 14 & 8 & 9 & - & 20 & 16 & 22 & 21 \\
26 & 1 & 22 & 24 & 13 & - & 16 & 14 & 12 \\
8 & 6 & 9 & 23 & 6 & 13 & - & 11 & 11 \\
9 & 2 & 14 & 20 & 18 & 16 & 11 & - & 4 \\
8 & 7 & 11 & 22 & 27 & 17 & 8 & 12 & -
\end{array}\right)
$$

The current cost $u_{c u r}^{w}$ for each passenger group $w \in W$ is defined by the matrix:

$$
U^{C U R}=\left(\begin{array}{ccccccccc}
- & 1.6 & 0.8 & 2 & 1.6 & 2.5 & 3 & 2.5 & 0.8 \\
2 & - & 0.9 & 1.2 & 1.5 & 2.5 & 2.7 & 2.4 & 1.8 \\
1.5 & 1.4 & - & 1.3 & 0.9 & 2 & 1.6 & 2.3 & 0.9 \\
1.9 & 2 & 1.9 & - & 1.8 & 2 & 1.9 & 1.2 & 2 \\
3 & 1.5 & 2 & 2 & - & 1.5 & 1.1 & 1.8 & 1.7 \\
2.1 & 2.7 & 2.2 & 1 & 1.5 & - & 0.9 & 0.9 & 2.9 \\
2.8 & 2.3 & 1.5 & 1.8 & 0.9 & 0.8 & - & 1.3 & 2.1 \\
2.8 & 2.2 & 2 & 1.1 & 1.5 & 0.8 & 1.9 & - & 0.3 \\
1 & 1.5 & 1.1 & 2.7 & 1.9 & 1.8 & 2.4 & 3 & -
\end{array}\right)
$$

The other network is a real network previously studied by Marín (2007). This network is denoted as Seville's network. It has 24 potential stations, 264 arcs, 552 passenger groups and a total demand of 292,000 passengers. This network is depicted in Fig. 2.

We present two different case studies in the following subsections. In Sect. 4.1, we evaluate our formulation without transfers (i.e., the IRTNDM) as compared to the classic formulation. We show how the improved formulation has a better linear programming relaxation, which improves the solution process and allows to solve bigger instances. In Sect. 4.2, we account for passengers' transfers at stations using the ITRTNDM. Consequently, the line topology 


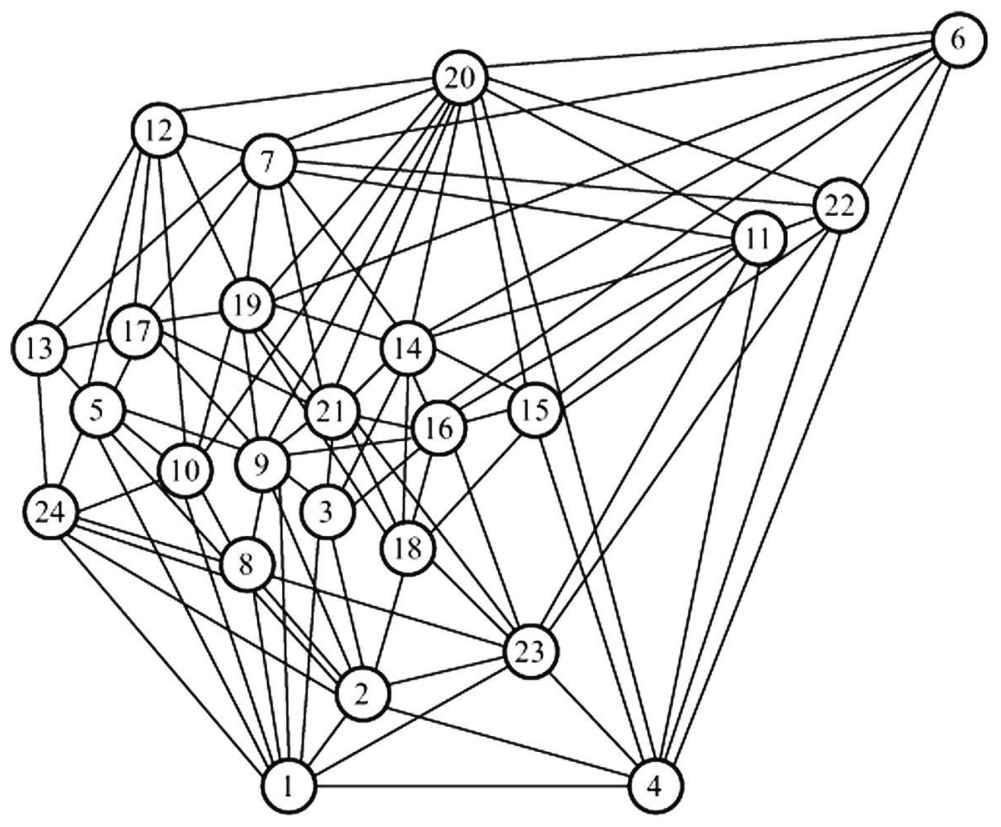

Fig. 2 Seville's network

will be developed minimizing the number of passengers that have to perform transfers to reach their destination.

For the computational experiments in this section we use the values for the weights in the objective function given by Marín and Jaramillo (2008). $\alpha$ is a number close to 1 , because demand coverage is the main component of the objective function. The other terms $(\beta, \gamma)$ are included to adequately simulate the routing user behavior and the location of any facility, which is not free of $\operatorname{cost}\left(\beta=\frac{1-\alpha}{2}, \gamma=\frac{1-\alpha}{2}\right)$.

We used for our tests a personal computer with an Intel Core 2 Quad CPU at $2.83 \mathrm{GHz}$ and 8 GB of RAM, running under Windows 7 64-Bit, and we implemented the models in GAMS/Cplex 12.1.

\subsection{Evaluation and performance of the formulation}

Two of the main parameters in all the presented mathematical models are the congestion factor and the maximum allowable budget. Variations in these parameters will lead to different network designs. We solve the Rapid Transit Network Design Model (RTNDM) [i.e., the mathematical model in Marín and García (2007) and Marín (2007)] varying the congestion factor and maximum allowable budget in order to compare the obtained solutions with the ones obtained using the IRTNDM formulation. The aim of this sensitivity analysis is twofold: first, to evaluate our approach and second, to verify the robustness of the model formulation. Hence, two different sensitivity analysis are performed: on the congestion factor and on the maximum allowable budget. We impose a maximum computational time of $4 \mathrm{~h}$ for every case study. Recall that we consider neither transfer constraints nor transfer variables for the rest of this subsection. 
The congestion factor $\mu_{w}$ is a parameter that calibrates the cost of traveling for each passenger group $w$ in the current network. We conduct seven different experiment runs for the sensitivity analysis on the congestion factor for each of the networks: network R1 and Seville's network. Budgets of 50 and 5000 are available for R1 and Seville's networks, respectively.

Table 1 shows the results for the sensitivity analysis on the congestion factor for network R1. We solve the RTNDM and the IRTNDM for the following congestion factors: $\epsilon \mu_{w}^{b}$ where $\epsilon$ takes the following values $0.9,1,1.1,1.2,1.3,1.4,1.5$ and $\mu_{w}^{b}$ is the base value for the congestion parameter for each passenger group. The heading of the table shows $\epsilon$ values in each column. Each row in the table displays a different item: the objective function $(z)$, the objective function of the linear relaxation of the problem $\left(z_{L P}\right)$, the demand attended in the new network $\left(g^{n e w}\right)$, the location cost $\left(z_{l o c}\right)$, the sum of the demand routing costs per passenger group in the new network $\left(z_{\text {route }}^{\text {new }}\right)$, the sum of the demand routing costs per passenger group in the current network $\left(z_{\text {route }}^{\text {cur }}\right)$, the percent of relative optimality gap (Gap), the computational time in seconds (Time) and the number of analyzed nodes in the Branch \& Bound algorithm (Nodes). In order to display the solutions provided by the two models, namely the RTNDM and the IRTNDM, each element of the table is composed of either one or two numbers: when the value provided by the models for the same item is identical, the element in the table displays a unique number; otherwise, it displays two numbers separated by a slash (i.e., 10/20: 10 corresponds to the solution provided by the RTNDM and 20 to the IRTNDM).

We must note that the objective function of the linear relaxation $\left(z_{L P}\right)$ of the IRTNDM is always greater than the linear relaxation of the RTNDM (it was at least a $70 \%$ greater for every test). This implies that the Branch \& Bound performance will be better and less effort will be needed to reach the optimum. Indeed, regarding the computational time (Time), we see that it is much lower for the IRTNDM; we could even say that it is near real-time for this network. Similarly, the number of analyzed nodes in the Branch \& Bound algorithm (Nodes) remains very low for the IRTNDM, as we expected. The rest of the output is the same for both formulations, thus we are able to obtain the same solution with significantly less effort.

Table 2 shows the results for the sensitivity analysis on the congestion factor for Seville's network. The results are very similar to those in Table 1 . We must note that the RTNDM almost always failed to prove optimality within the available computational time. For congestion factor values corresponding to $\epsilon$ equal to 1.1 and 1.3 both formulations failed. However, there are differences here: first, the optimality gap was significantly lower for the IRTNDM when time was over (very close to 0 ), and second, computational times needed by the IRTNDM to reach $1 \%$ of relative optimality gap were 210 and $250 \mathrm{~s}$ for congestion factors corresponding to $\epsilon$ equal to 1.1 and 1.3 , respectively.

The maximum available budget is another parameter that highly conditions the design of the network. Therefore, we conduct again different experiment runs and compare the solutions provided by the IRTNDM with the solutions provided by the RTNDM. This sensitivity analysis is conducted on two different networks: network R1 and Seville's network. For these experiments $\epsilon$ is assumed to be 1.2 .

Table 3 shows the results for the sensitivity analysis on the maximum available budget for the network R1. We solve the RTNDM and the IRTNDM for the following maximum available budgets: $10,20,25,30,40,50$. The heading of the table shows these budget values in each column. Each row in the table displays a different item: the objective function $(z)$, the demand attended in the new network $\left(g^{\text {new }}\right)$, the location cost $\left(z_{l o c}\right)$, the sum of the demand routing costs per passenger group in the new network $\left(z_{\text {route }}^{\text {new }}\right)$, the sum of the demand routing 
Table 1 Results of the RTNDM and IRTNDM for the sensitivity analysis on the congestion factor for network R1; when the table displays a unique number the values provided by the RTNDM and IRTNDM are the same; otherwise, the values are separated by a slash (RTNDM/IRTNDM)

\begin{tabular}{|c|c|c|c|c|c|c|c|}
\hline Item $\backslash \epsilon$ & 0.9 & 1 & 1.1 & 1.2 & 1.3 & 1.4 & 1.5 \\
\hline$z$ & 440.9 & 414.2 & 395.5 & 393.2 & 383.7 & 382.1 & 386.5 \\
\hline$z_{L P}$ & $220 / 381.8$ & $201.7 / 348.4$ & $175.8 / 307.7$ & $169.1 / 287.4$ & $150.9 / 265.1$ & $139 / 248.6$ & $138.9 / 238.9$ \\
\hline$g^{\text {new }}$ & 764 & 796 & 823 & 830 & 844 & 850 & 850 \\
\hline zloc & 49.9 & 49.9 & 49.5 & 49.5 & 49.5 & 49.5 & 49.5 \\
\hline$z_{\text {route }}^{\text {new }}$ & 61.5 & 67.3 & 72.9 & 74.2 & 75.6 & 77.6 & 77.6 \\
\hline$z_{\text {route }}^{\text {cur }}$ & 49.5 & 49 & 52.1 & 55.5 & 58.6 & 61 & 65.4 \\
\hline Gap & 0 & 0 & 0 & 0 & 0 & 0 & 0 \\
\hline Time (s) & $6442.3 / 0.7$ & $5895 / 0.7$ & $7367.8 / 0.9$ & $8191 / 1.4$ & $6151.8 / 1.5$ & $4984.4 / 1.3$ & $10,395.4 / 2.2$ \\
\hline Nodes & $1,110,000 / 269$ & $759,000 / 182$ & $888,000 / 1$ & $1,130,000,000 / 223$ & $1,060,000 / 1$ & $595,000 / 1$ & $1,290,000,000 / 445$ \\
\hline
\end{tabular}


Table 2 Results of the RTNDM and IRTNDM for the sensitivity analysis on the congestion factor for Seville's network; when the table displays a unique number the values provided by the RTNDM and IRTNDM are the same; otherwise, the values are separated by a slash (RTNDM/IRTNDM)

\begin{tabular}{|c|c|c|c|c|c|c|c|}
\hline Item $\backslash \epsilon$ & 0.9 & 1 & 1.1 & 1.2 & 1.3 & 1.4 & 1.5 \\
\hline$z$ & 3.17E5 & $2.51 \mathrm{E} 5$ & $1.06 \mathrm{E} 5 / 75,751.4$ & $67,320 / 56,046.3$ & $48,153.5 / 48,128.5$ & $48,173.1 / 48,130.2$ & $47,679 / 47,658.5$ \\
\hline$z_{L P}$ & $3.16 \mathrm{E} 5 / 3.17 \mathrm{E} 5$ & 2.3E $5 / 2.51 \mathrm{E} 5$ & $48,060.5 / 71,951.4$ & $45,987.3 / 53,774.1$ & $45,978.7 / 48,099.2$ & $46,051.3 / 48,126.7$ & $45,582 / 47,657$ \\
\hline$g^{\text {new }}$ & 2306 & $74,872 / 75,262$ & $2.21 \mathrm{E} 5 / 2.52 \mathrm{E} 5$ & $2.61 \mathrm{E} 5 / 2.72 \mathrm{E} 5$ & $2.80 \mathrm{E} 5 / 2.80 \mathrm{E} 5$ & $2.80 \mathrm{E} 5 / 2.80 \mathrm{E} 5$ & $2.81 \mathrm{E} 5 / 2.81 \mathrm{E} 5$ \\
\hline zloc & 798.2 & $4993.3 / 4997.1$ & $4997.9 / 4991.7$ & $4993.1 / 4993.3$ & $4986.5 / 4996.4$ & $4996 / 4970.8$ & $4959.5 / 4939.1$ \\
\hline$z_{\text {roule }}^{\text {new }}$ & 66.8 & $3057.9 / 2899.9$ & $22,671.5 / 25,562$ & $27,405.7 / 28,073.5$ & $29,282.4 / 29,247.7$ & $29,206.6 / 29,188.9$ & $29,222.3 / 29,222.3$ \\
\hline$z_{\text {route }}^{\text {cur }}$ & $25,676.7$ & $25,523.3 / 25,687$ & $7513.7 / 4373.5$ & $3638.1 / 2240.4$ & $1118.3 / 1118.3$ & $1204.4 / 1204.4$ & $1284 / 1284$ \\
\hline Gap & $0 / 0$ & $4.1 / 0$ & $53.4 / \approx 0$ & $30.2 / 0$ & $2.5 / \approx 0$ & $2.2 / 0$ & $2 / 0$ \\
\hline Time (s) & $142.9 / 15$ & $14,401.5 / 50.7$ & $14,400.5 / 14,400.5$ & $14,413.3 / 830.3$ & $14,400.5 / 14,400.5$ & $14,400.8 / 139$ & $14,400.8 / 75$ \\
\hline Nodes & $506 / 1$ & $140,000 / 1073$ & $35,300 / 1,070,000$ & $27,370 / 11,629$ & $49,800 / 558,000$ & $34,243 / 513$ & $49,720 / 510$ \\
\hline
\end{tabular}


Table 3 Results of the RTNDM and IRTNDM for the sensitivity analysis on the maximum available budget for network R1; when the table displays a unique number the values provided by the RTNDM and IRTNDM are the same; otherwise, the values are separated by a slash (RTNDM/IRTNDM)

\begin{tabular}{|c|c|c|c|c|c|c|}
\hline Item $\backslash$ budget & 10 & 20 & 25 & 30 & 40 & 50 \\
\hline$z$ & 1227.7 & 1146.1 & 1001 & 899.3 & 730 & 393.2 \\
\hline$g^{n e w}$ & 56 & 137 & 225 & 368 & 525 & 830 \\
\hline$z_{l o c}$ & 9.6 & 17.8 & 23.8 & 29.2 & 37.3 & 49.5 \\
\hline$z_{\text {route }}^{\text {new }}$ & 1.4 & 7.2 & 13.2 & 22 & 36 & 74.2 \\
\hline$z_{\text {route }}^{\text {cur }}$ & 228.7 & 214.1 & 145 & 172.1 & 137.7 & 55.5 \\
\hline Gap & 0 & 0 & 0 & 0 & 0 & 0 \\
\hline Time (s) & $0.9 / 0.7$ & $108.2 / 5.1$ & $432.4 / 2.7$ & $433.7 / 4.9$ & $9826 / 10.8$ & $8191 / 1.4$ \\
\hline Nodes & $73 / 3$ & $9956 / 1045$ & $49,322 / 1266$ & $97,047 / 904$ & $2,080,000,000 / 8047$ & $1,130,000,000 / 223$ \\
\hline
\end{tabular}


costs per passenger group in the current network $\left(z_{\text {route }}^{\text {cur }}\right)$, the percent of relative optimality gap (Gap), the computational time in seconds (Time) and the number of analyzed nodes in the Branch \& Bound algorithm (Nodes). The information displayed in this table follows the same scheme as before. Again, the computational time (Time) is much lower for the IRTNDM and the number of analyzed nodes in the Branch \& Bound algorithm (Nodes) remains very low for the IRTNDM, thus we are able to obtain the same solution with significantly less effort.

Table 4 shows the results for the sensitivity analysis on the maximum available budget for Seville's network. We solve the RTNDM and the IRTNDM for the following maximum available budgets: $1000,2000,3000,5000,10,000$. The information displayed in this table is the same as in Table 3. We must note that the RTNDM failed to demonstrate optimality for all the cases studied in this sensitivity analysis: the relative optimality gap ranged from the $3.3 \%$ to the $76.52 \%$, which is significantly far from the optimum. However, the IRTNDM solved almost all the cases to the optimum and for those which did not, the relative optimality gap was lower than $1 \%$.

\subsection{Impacts of considering transfers}

Passengers choose the most convenient routes and modes in order to carry out their trips. A decisive factor for attracting passengers to the new network is to offer direct trips without transfers. In order to show the benefits of considering passengers' transfers we conduct computational experiments on the previously introduced networks. García et al. (2006) are among the first to study transfers in rapid transit network design problems. However, their approach cannot solve medium- or large-scale problems. Computational time was high for small-scale problems.

The TRTNDM has a weak linear relaxation and Branch \& Bound fails to find the optimum (or even a feasible solution) for any of the proposed case studies. Therefore, we directly solve the ITRTNDM. Table 5 shows the results for network R1. We solve the IRTNDM and the ITRTNDM for the following values of $\epsilon: 0.9$ and 1; the heading of the table shows these $\epsilon$ values. Each row in the table displays a different item; they can be read the same way as in the previous tables (there are two new rows: $c_{\max }$, the maximum available budget and, Transfers, the number of transfers made by the passengers). In order to display the solutions provided by the two variants of the model, namely the IRTNDM and the ITRTNDM, each element of the table is composed of either one or two numbers: when the value provided by the models for the same item is identical, the element in the table displays a unique number otherwise it displays two numbers separated by a slash (i.e., 10/20: 10 corresponds to the solution provided by the IRTNDM and 20 to the ITRTNDM), otherwise.

We must note that the number of transfers is reduced for every computational experiment in the network R1. This is always a desirable issue because offering direct trips is always a positive factor for attracting passengers. The attended demand in the new network $\left(g^{\text {new }}\right)$ is the same for all the case studies in the network $\mathrm{R} 1$. The routing cost in the new network $\left(z_{\text {route }}^{\text {new }}\right)$ was greater almost for all the cases in the solutions provided by the ITRTNDM: this is due to the fact we are including a transfer cost for passengers. As one may expect, the ITRTNDM is always more difficult to be solved: the computational effort in terms of time is greater than the one of the IRTNDM.

Table 6 shows the same information as Table 5 but for Seville's network. Here, the main objective of reducing the number of transfers is again reached. 
Table 4 Results of the RTNDM and IRTNDM for the sensitivity analysis on the maximum available budget for Seville's network; when the table displays a unique number the values provided by the RTNDM and IRTNDM are the same; otherwise, the values are separated by a slash (RTNDM/IRTNDM)

\begin{tabular}{|c|c|c|c|c|c|}
\hline Item $\backslash$ budget & 1000 & 2000 & 3000 & 5000 & 10,000 \\
\hline$z$ & $3.10 \mathrm{E} 5 / 3.06 \mathrm{E} 5$ & $2.75 \mathrm{E} 5 / 2.52 \mathrm{E} 5$ & $2.18 \mathrm{E} 5 / 1.82 \mathrm{E} 5$ & $67,320 / 56,046.3$ & $48,500.5 / 48,362.6$ \\
\hline$g^{n e w}$ & $18,106 / 21,555$ & $53,356 / 76,605$ & $1.11 \mathrm{E} 5 / 1.46 \mathrm{E} 5$ & $2.62 \mathrm{E} 5 / 2.72 \mathrm{E} 5$ & $2.80 \mathrm{E} 5 / 2.80 \mathrm{E} 5$ \\
\hline$z_{l o c}$ & $971 / 989.3$ & $1998.9 / 1986.4$ & $2983.7 / 2994.4$ & $4993.1 / 4993.3$ & $6002.3 / 5788$ \\
\hline$z_{\text {route }}^{\text {new }}$ & $1578.3 / 2225.9$ & $5416.7 / 8556.9$ & $9945.2 / 14,794$ & $27,405.7 / 28,073.5$ & $28,699.8 / 28,776.2$ \\
\hline$z_{\text {route }}^{\text {cur }}$ & $32,457 / 31,764.9$ & $28,223.1 / 24,696.4$ & $23,015.3 / 17,539.3$ & $3638.1 / 2240.4$ & $1032.3 / 1032.3$ \\
\hline Gap & $3.30 / 0$ & $70.46 / 0.83$ & $76.52 / 0$ & $30.45 / 0$ & $3.30 / 0.04$ \\
\hline Time (s) & $14,400.6 / 12,706$ & $14,400.6 / 14,400.6$ & $14,400.6 / 4091.1$ & $14,413.3 / 830.3$ & $14,419.8 / 14,400.6$ \\
\hline Nodes & $1983 / 21,951$ & $2495 / 40,200$ & $6181 / 14,719$ & $27,370 / 11,629$ & $55,323 / 1,650,000,000$ \\
\hline
\end{tabular}


Table 5 Results of the IRTNDM and ITRTNDM for network R1; when the table displays a unique number the values provided by the IRTNDM and ITRTNDM are the same; otherwise, the values are separated by a slash (IRTNDM/ITRTNDM)

\begin{tabular}{lllll}
\hline Item $\backslash \epsilon$ & 0.9 & & 1 & \\
\hline$c_{\text {max }}$ & 25 & 50 & 25 & 50 \\
$z$ & $1001 / 1001.4$ & $440.9 / 443.3$ & 1015.4 & $414.2 / 417.2$ \\
$g^{\text {new }}$ & 225 & 764 & 233 & 796 \\
$z_{\text {loc }}$ & $23.8 / 23$ & 49.9 & 23.9 & 49.9 \\
$z_{\text {route }}^{\text {new }}$ & $13.2 / 14.4$ & $61.5 / 63.9$ & 14.7 & $67.3 / 70.3$ \\
$z_{\text {route }}^{\text {cur }}$ & 145 & 49.5 & 165.8 & 49 \\
Transfers & $97 / 0$ & $460 / 250$ & $56 / 0$ & $545 / 340$ \\
Gap & 0 & 0 & 0 & 0 \\
Time $(\mathrm{s})$ & $0.7 / 8.2$ & $0.7 / 10.8$ & $3.9 / 13.1$ & $0.7 / 16.3$ \\
Nodes & $618 / 219$ & $269 / 111$ & $1333 / 222$ & $182 / 423$ \\
\hline
\end{tabular}

Table 6 Results of the IRTNDM and ITRTNDM for Seville's network; when the table displays a unique number the values provided by the IRTNDM and ITRTNDM are the same; otherwise, the values are separated by a slash (IRTNDM/ITRTNDM)

\begin{tabular}{lllll}
\hline Item $\backslash \epsilon$ & 0.9 & & 1 & \\
\hline$c_{\text {max }}$ & 5000 & 10,000 & 5000 & 10,000 \\
$z$ & $3.17 \mathrm{E} 5$ & $3.17 \mathrm{E} 5$ & $2.51 \mathrm{E} 5 / 2.52 \mathrm{E} 5$ & $2.29 \mathrm{E} 5 / 2.30 \mathrm{E} 5$ \\
$g^{\text {new }}$ & 2306 & 2306 & $75,262 / 74,260$ & $99,509 / 98,671$ \\
$z_{\text {loc }}$ & 798.2 & 798.2 & $4997.1 / 4956.6$ & $7517.2 / 7653.8$ \\
$z_{\text {rowte }}^{\text {new }}$ & 66.8 & 66.8 & $2899.9 / 2918.4$ & $5328.4 / 5301.2$ \\
$z_{\text {route }}^{\text {cur }}$ & $25,676.7$ & $25,676.7$ & $25,687 / 25,663.5$ & $23,252.6 / 23,282.8$ \\
Transfers & 0 & 0 & $3796 / 3252$ & $5830 / 2306$ \\
Gap & 0 & 0 & $0 / 0.99$ & $0 / 0.45$ \\
Time $(s)$ & $14.3 / 58.2$ & $13.3 / 57.4$ & $43.1 / 14,402.1$ & $45.1 / 14,402.1$ \\
Nodes & $1 / 363$ & $1 / 204$ & $1073 / 1908$ & $426 / 3914$ \\
\end{tabular}

\section{Conclusions and further research}

We have studied the problem of designing rapid transit networks which consists of locating stations and alignments between them. Here, the current and the new network to be constructed must be considered in order to maximize the coverage of the new network which is competing against the current one.

We have proposed a formulation for the rapid transit network design problem. This formulation is a stronger formulation as compared to previously developed formulations because it is able to provide tighter linear relaxation values. Therefore, we are able to solve real-case studies within reasonable computational times using exact optimization methods such as Branch \& Bound.

The improvement in the formulation allows us to introduce new features in the problem. We explicitly consider the transfers of passengers. This is a very important attribute the passenger 
demand will consider when choosing its means of transport. Consequently, minimizing the number of passengers that must perform a transfer to reach destination is highly desirable.

We have conducted computational experiments on two different networks. One of them is a realistic case drawn form the city of Seville in Spain. We have conducted sensitivity analysis on two model parameters: the congestion and the maximum allowable budget. The former measures the expected level of congestion of the current network and the latter the available budget for constructing the new network. We have shown that our formulation is robust in the sense it always provides optimal or near-optimal solutions.

In our future research we are going to embark on a study of robust solutions. Further research needs to refine the concept of robustness when applied to network design problems. For this purpose the recoverable robustness will be studied. Also, more complex demand behavior models will be addressed.

\section{Compliance with ethical standards}

Conflict of interest The authors declare that they have no conflict of interest.

\section{References}

Bruno, G., Gendreau, M., \& Laporte, G. (2002). A heuristic for the location of a rapid transit line. Computers \& Operations Research, 29, 1-12.

Bruno, G., Ghiani, G., \& Improta, G. (1998). A multi-modal approach to the location of a rapid transit line. European Joumal of Operational Research, 104, 321-332.

Bruno, G., \& Laporte, G. (2002). An interactive decision support system for the design of rapid public transit networks. Information Systems and Operational Research, 40, 111-118.

Cadarso, L., \& Marín, Á. (2010). Robust routing of rapid transit rolling stock. Public Transport, 2(1-2), 51-68.

Cadarso, L., \& Marín, Á. (2011). Robust rolling stock in rapid transit networks. Computers \& Operations Research, 38(8), 1131-1142.

Cadarso, L., \& Marín, Á. (2012). Integration of timetable planning and rolling stock in rapid transit networks. Annals of Operations Research, 199(1), 113-135.

Dufourd, H., Gendreau, M., \& Laporte, G. (1996). Locating a transit linen using Tabu search. Location Science, 4, 1-19.

Escudero, L. F., \& Muñoz, S. (2009). An approach for solving a modification of the extended rapid transit network design problem. Top, 17(2), 320-334.

Fan, W., Gendreau, M., \& Machemehl, R. B. (2006). Using a simulated annealing algorithm to solve the transit route network design problem. Joumal of Transportation Engineering, 132, 122-132.

García, R., Garzón-Astolfi, A., Marín, A., Mesa, J. A., \& Ortega, F.A. (2006). Analysis of the parameters of transfers in the rapid transit network design. Schloss Dagstuhl. http://drops.dagstuhl.de/opus/volltexte/ $2006 / 658$.

Gendron, B., \& Crainic, T.G. (1994). Relaxations for multicommodity capacitated network design problems. Publication CRT-965, Centre de recherche sur les transpont, Montreal.

Gendron, B., Crainic, T. G., \& Frangioni, A. (1999). Telecomunication network planning, chapter multicommodity capacitated network design. Dordrecht: Academic.

Gutiérrez-Jarpa, G., Obreque, C., Laporte, G., \& Marianov, V. (2013). Rapid transit network design for optimal cost and origin-destination demand capture. Computers \& Operations Research, 40(12), 3000-3009.

Hamacher, H., Liebers, A., Schobel, A., Wagner, D., \& Wagner, F. (2001). Locating new stops in a railway network. Electronic Notes in Theoretical Computer Science, 50, 153-178.

Kermansshahi, S., Shafahi, M., Mollanejad, Y., \& Zangui, M. (2010). Rapid transit network design using simulated annealinge. In 12 th World conference of transportation research, Lisbon, Portugal, July 1115.

Laporte, G., Marín, A., Mesa, J. A., \& Ortega, F. A. (2007). An integrated methodology for the rapid transit network design. Lecture Notes in Computationa Science, 4359, 187-199.

Laporte, G., Marín, A., Mesa, J. A., \& Ortega, F. A. (2011a). Designing robust rapid transit networks with alternative routes. Journal of Advanced Transportation, 45, 54-65. 
Laporte, G., Mesa, J. A., \& Ortega, F. A. (2000). Optimization methods for the planning of rapid transit systems. European Joumal of Operational Research, 122, 1-10.

Laporte, G., Mesa, J. A., Ortega, F. A., \& Perea, F. (2011b). Planning rapid transit networks. Socio-Economic Planning Sciences, 45, 95-104.

Laporte, G., Mesa, J. A., \& Ortega, F. A. (2002). Locating stations on rapid transit lines. Computers \& Operations Research, 29, 741-759.

Laporte, G., Mesa, J. A., \& Ortega, F. A. (2005). Maximizing trip coverage in the location of a single rapid transit alignment. Annals of Operations Research, 136, 49-63.

Marín, A. (2007). An extension to rapid transit network design problem. Top, 15, 231-241.

Marín, A., \& García, R. (2007). Location of the infrastructure in urban railway networks. Computer and Operations Research, 36, 1461-1477.

Marín, A., \& Jaramillo, P. (2008). Urban rapid transit network capacity expansion. European Joumal of Operation Research, 191, 45-60.

Ngamchai, S., \& Lovell, D. (2003). Optimal time transfer in bus transit route network design using a genetic algorithm. Joumal of Transportation Engineering, 129(5), 510-521.

Repolho, H. M., Antunes, A. P., \& Church, R. L. (2013). Optimal location of railway stations: The lisbon-porto high-speed rail line. Transportation Science, 47(3), 330-343.

Tom, V. M., \& Mohan, S. (2003). Transit route nework design using frequency coded genetic algorithm. Journal of Transportation Engineering, 129(2), 186-195.

Zhao, F., \& Ubaka, I. (2004). Transit nework optimization-minimizing transfer and optimazing route directness. Joumal of Transportation Engineering, 7(1), 67-82. 